\title{
Optimizing Stature in Congenital Adrenal Hyperplasia: Challenges and Solutions
}

\author{
Anju Seth ${ }^{1}$
}

Received: 9 April 2019 / Accepted: 9 April 2019 / Published online: 27 April 2019

(C) Dr. K C Chaudhuri Foundation 2019

Growth faltering is a frequent consequence of chronic disorders in children. Classical congenital adrenal hyperplasia $(\mathrm{CAH})$ is one such disorder where failure to reach age related norms and genetic potential in height has been consistently reported. Management of CAH requires life-long regular monitoring, and periodic dose adjustment of the two basic drugs viz. glucocorticoids (GC) and mineralocorticoids (MC). Inadequate doses of GC predispose the child to risk of salt wasting and excess androgenisation leading to virilisation in girls and sexual precocity in boys. Androgen excess also leads to advancement of bone age, leading to ultimate short stature due to early epiphyseal fusion. Excessive GC on the other hand, suppresses growth by direct effect on growth plate and disruption of growth hormone-insulin like growth factor-1 (GH-IGF1) axis at various levels [1]. Thus, one of the biggest therapeutic challenges in management of $\mathrm{CAH}$ is determining and ensuring compliance on a continuous basis, with the right dose of GC, that is sufficient to suppress excessive androgenisation, while providing adequate replacement and having minimum growth inhibitory effect. Many studies across the world have reported final height in patients with $\mathrm{CAH}$ to be less than the reference norms and the genetic potential [2-4].

In the current issue of the Journal, Meena et al. have reported on the growth pattern of patients with CAH under their care [5]. They observed a low growth velocity in more than half of the children, while $31 \%$ had short stature. The stature was more severely affected in children under $2 \mathrm{y}$ of age, all of whom had poor growth velocity, and in the adolescent. The height standard deviation score (SDS) were relatively better preserved in age group 2-10 y. As the authors surmised, the

Anju Seth

anjuseth.peds@gmail.com

1 Department of Pediatrics, Lady Hardinge Medical College, New Delhi, India cause of poor growth in younger children was likely due to salt wasting and relatively high doses of GC administered both prior to enrolment at their Institute and during follow-up. Short stature during adolescence was likely to be due to a combined effect of advanced bone age and early onset of puberty. The authors have not mentioned what proportion of their patients had a secondary central precocious puberty, that can complicate the course of boys with $\mathrm{CAH}$, who receive relatively lower doses of GC, with resultant inadequate androgen suppression and bone age advancement. The relatively well preserved stature during mid-childhood years should be interpreted with caution; it is very likely to represent an accelerated growth secondary to androgen excess, a possibility supported by the fact that maximum bone age advancement was also observed in this age group. This accelerated growth in mid-childhood years would in fact be a major contributor to ultimate short stature in these children. The mean final height SDS score among 6 children who had attained adult stature was -2 . A factor contributing towards short stature in patients in this study is delayed diagnosis in males with simple virilising $\mathrm{CAH}$ who are detected only once they develop features of androgen excess, by which time they would have significant advancement of bone age. Yet another factor is limited availability of pediatric endocrine services in the country that are critical for providing appropriate long term care for this chronic disorder. This leads to a delay in diagnosis and suboptimal management unless timely referral occurs to a centre where such expertise exists. Another recent Indian study has reported a mean final height SDS of $-2.06 \pm 1.1$ for boys and $-1.47 \pm 1.1$ for girls with $\mathrm{CAH}[6]$.

The following strategies can help improve the height outcome in this difficult to manage disorder:

1. High index of suspicion and early diagnosis: Suggestive features include clitoromegaly in female infants, failure to thrive and hyponatremia in infants of either sex, and sexual precocity with dark pigmentation in boys during early childhood. 
2. Timely enrolment in follow-up care at a centre where expertise in management exists.

3. Meticulous clinical follow-up: The Endocrine Society, USA, recommends a close monitoring during first $3 \mathrm{mo}$, a 3 monthly follow-up from 3 to 18 mo and 4 monthly thereafter [7]. Monitoring should include assessment of weight, height, blood pressure, pubertal development and features of glucocorticoid/ androgen excess. Accelerated height velocity, signs of virilization, and advanced bone maturation indicate protracted under-treatment, while reduced height velocity, accelerated weight gain, and high blood pressure suggest protracted overtreatment.

4. Periodic laboratory monitoring: This includes measuring serum electrolytes, 17-hydroxy progesterone (17OHP), androstenedione and plasma renin activity (PRA) if feasible, every 3 monthly in infancy and 412 monthly thereafter [8]. The aim is to maintain serum $17 \mathrm{OHP}$ between 5 and $10 \mathrm{ng} / \mathrm{ml}$ and not to achieve age specific normal level - that would require substantial overdosing with its associated adverse impact on height. Serum androstenedione should be maintained as per age and sex recommended norms. Testosterone may be measured instead of androstenedione in girls and pre-pubertal boys. Bone age is estimated annually. The GC and MC dose is carefully adjusted by taking in account the body surface area, height velocity, weight gain, blood pressure (BP), bone age along with biochemical parameters.

5. Use of hydrocortisone over more potent GC: Prednisolone has a 15-fold and dexamethasone 70-80fold higher growth inhibitory effect as compared to hydrocortisone $[9,10]$. Thus, it is recommended that hydrocortisone be used as a GC of choice, at-least till the growth of the child is completed.

6. Administration of fludrocortisone to all children with $\mathrm{CAH}$, since sub-clinical aldosterone deficiency is present in all children with classical CAH including those who have the simple virlising form. Maintaining sodium balance achieves euvolemia, reduces adrenocorticotropic hormone (ACTH) levels and hence helps reduce GC dose required [2].

7. Early detection and management of central precocious puberty by use of gonadotrophin releasing hormone ana$\operatorname{logs}[11]$.

8. Judicious use of growth hormone and newer steroid sparing strategies: Growth hormone alone or along with gonadotrophin releasing hormone analogs may be used to augment height in patients expected to be significantly short [12]. Newer therapies that improve height potential by decreasing the GC dose include anti-androgens and aromatase inhibitors [13].

9. Last, but not the least, early detection of CAH by neonatal screening is invaluable especially in males with simple virilising forms who often escape detection till a variable age, while also being lifesaving for both sexes with salt loosing forms.

To summarise, an early diagnosis, timely referral to a centre with management expertise, meticulous monitoring with careful, ongoing adjustment in GC and MC dose and judicious use of growth enhancing strategies in selected cases can help optimise the growth potential in children with $\mathrm{CAH}$.

\section{Compliance with Ethical Standards}

Conflict of Interest None.

\section{References}

1. Hochberg Z. Mechanisms of steroid impairment of growth. Horm Res. 2002;58:33-8.

2. Muthusamy K, Elamin MB, Smushkin G, et al. Clinical review: adult height in patients with congenital adrenal hyperplasia: a systematic review and meta-analysis. J Clin Endocrinol Metab. 2010;95:4161-72.

3. Bonfig W. Growth and development in children with classic congenital adrenal hyperplasia. Curr Opin Endocrinol Diabetes Obes. 2017;24:39-42.

4. Alzanbagi MA, Milyani AA, Al-Agha AE. Growth characteristics in children with congenital adrenal hyperplasia. Saudi Med J. 2018;39:674-8.

5. Meena H, Jana M, Singh V, Kabra M, Jain V. Growth pattern and clinical profile of Indian children with classical 21-hydroxylase deficiency congenital adrenal hyperplasia on treatment. Indian J Pediatr. 2019. https://doi.org/10.1007/s12098-018-02848-6.

6. Maheshwari A, Khadilkar V, Gangodkar P, Khadilkar A. Long-term growth in congenital adrenal hyperplasia. Indian J Pediatr. 2019;86: 154-8.

7. Speiser PW, Arlt W, Auchus RJ, et al. Congenital adrenal hyperplasia due to steroid 21-hydroxylase deficiency: an Endocrine Society Clinical Practice Guideline. J Clin Endocrinol Metab. 2018;103:4043-88.

8. Joint LWPES/ESPE CAH Working Group. Consensus statement on 21-hydroxylase deficiency from the Lawson Wilkins Pediatric Endocrine Society and the European Society for Paediatric Endocrinology. J Clin Endocrinol Metab. 2002;87:4048-53.

9. Rivkees SA, Crawford JD. Dexamethasone treatment of virilising congenital adrenal hyperplasia: the ability to achieve normal growth. Pediatrics. 2000;106:767-73.

10. Punthakee Z, Legault L, Polychronakos C. Prednisolone in the treatment of adrenal insufficiency: a re-evaluation of relative potency. J Pediatr. 2003;143:402-5.

11. Güven A, NurcanCebeci A, Hancili S. Gonadotropin releasing hormone analog treatment in children with congenital adrenal 
hyperplasia complicated by central precocious puberty. Hormones (Athens). 2015;14:265-71.

12. Quintos JBQ, Vogiatzi MG, Harbison MD, New MI. Growth hormone therapy alone or in combination with onadotropin releasing hormone analog therapy to improve the height deficit in children with congenital adrenal hyperplasia. J Clin Endocrinol Metab. 2001;86:1511-7.
13. Laue L, Merke DP, Jones JV, Barnes KM, Hill S, Cutler GB Jr. A preliminary study of flutamide, testolactone, and reduced hydrocortisone dose in the treatment of congenital adrenal hyperplasia. J Clin Endocrinol Metab. 1996;81:3535-9.

Publisher's Note Springer Nature remains neutral with regard to jurisdictional claims in published maps and institutional affiliations. 